\title{
Enabling Solution Growth of Insoluble Organic Materials in Common Solvents
}

\author{
Masaaki Yokota1* $^{*}$ Kota Fujii1, Makoto Ishigo', Kuniaki Sasaki', Hitoshi Kato², \\ Norihito Doki 1 \\ ${ }^{1}$ Department of Chemistry and Bioengineering, Iwate University, Morioka, Japan \\ ${ }^{2}$ Tokyo Electron Tohoku Limited, Iwate, Japan \\ Email: "myokota@iwate-u.ac.jp
}

Received 25 December 2015; accepted 5 April 2016; published 8 April 2016

Copyright (C) 2016 by authors and Scientific Research Publishing Inc.

This work is licensed under the Creative Commons Attribution International License (CC BY). http://creativecommons.org/licenses/by/4.0/

c) (i) Open Access

\begin{abstract}
Copper phthalocyanine (CuPc) amorphous film was successfully deposited on a silicone substrate by physical vapor deposition. When the film was in contact with a common solvent such as aniline, 1-propanol and toluene, the CuPc solid film was partially dissolved followed by nucleation and crystal growth in the solution. Based on these experimental results, we propose a novel method for preparation of the organic thin film by combination of dry and wet processes.
\end{abstract}

\section{Keywords}

Crystallization, Amorphous, Copper Phthalocyanine, Organic Thin Film

\section{Introduction}

Variety of organic and/or organometallic compounds have been designed and synthesized for application of electronic devices [1] [2]. For development of the electronic materials, design of the organic molecules with required electronic properties is important and many organic chemists have been synthesized variety of the compounds [1] [2]. In addition to the importance of the molecular design, control of the molecular assembly is also important so as to maximize the potential properties of the molecules. In controlling the molecular packing and/or assembly manner, it is very useful to take advantage of the character of crystals. As well known, molecules are packed in a regular manner with periodic structures in crystals. Thus we want to strongly say that if one may control nucleation and crystal growth on the film, quality of the thin film must be enhanced. It is very important to control nucleation and crystal growth during the organic film deposition. There exists two methods, dry and wet processes, for preparation of organic films. Both methods have advantages and disadvantages. How-

${ }^{*}$ Corresponding author.

How to cite this paper: Yokota, M., Fujii, K., Ishigo, M., Sasaki, K., Kato, H. and Doki, N. (2016) Enabling Solution Growth of Insoluble Organic Materials in Common Solvents. Advances in Chemical Engineering and Science, 6, 82-86.

http://dx.doi.org/10.4236/aces.2016.62010 
ever, wet process progresses relatively slower than dry process and the wet process must be suitable to control crystallization. Unfortunately, in most organic materials used for electronic devices, molecules have structures that are difficult to be dissolved in solvents. This makes the application of wet processes difficult. In this paper, we propose a simple method for achieving the dissolution of insoluble organic chemicals even in common solvents and an evidence of crystallization of such compound has been shown. The target molecule employed in this paper is copper phthalocyanine (CuPc).

\section{Experimental}

\subsection{Materials}

Copper phthalocyanine, (CuPc, $>90.0 \%$, Figure 1) used in this study was purchased from Tokyo Chemical Industry. As common solvents, we used, aniline (Tokyo Chemical Industry, >98.0\%), 1-propanol (Tokyo Chemical Industry, >99.5\%), toluene (Wako Pure Chemical Industries, 99.0\%). These materials were used without purification.

\subsection{Method}

Our methodology proposes in this study is composed of two steps. At first, CuPc is solidificated as unstable solid film (amorphous) in order to promote the dissolution. Using the amorphous film, crystallization of the accumulated CuPc in solution is challenged.

Preparation of amorphous CuPc film was done as follows using physical vapor deposition equipment (Figure 2), usually used for pre-treatment of SEM samples. CuPc powder samples were put on the sample folder and the temperature of the samples were elevated by resistance heating method in vacuo (about $6 \times 10^{-4} \mathrm{~Pa}$ ). On heating, sublimation of the sample was occurred and the CuPc was deposited onto the substrate. Deposited CuPc film was observed using SEM, AFM and was characterized by XRD.

In the next wet process, a droplet of the solvent was put on the CuPc film made by the first dry stage and changes in the solid state was observed. In this wet process, samples were allowed to set in an open air at room temperature.

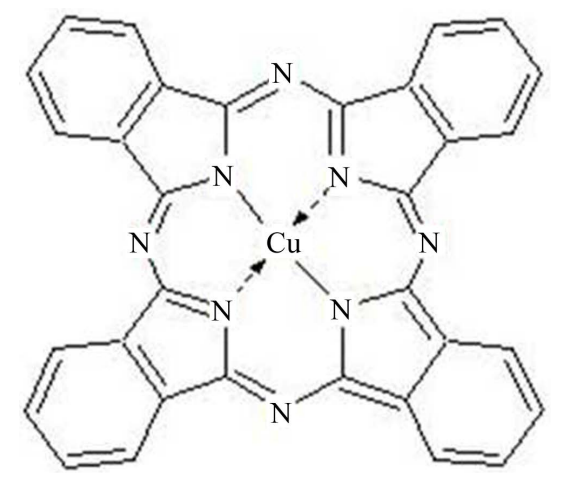

Figure 1. Chemical structure of Copper phthalocyanine $(\mathrm{CuPc})$.

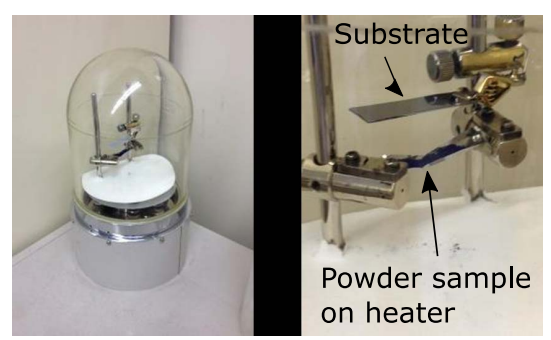

Figure 2. Photographic pictures of physical vapor deposition equipment. 


\section{Results and Discussion}

\subsection{CuPc Vapor Deposition in Dry-Process}

Typical example of physical vapor deposition of CuPc onto the silicone substrate is shown in Figures 3(a)-(d). The color of the substrate was changed to blue after vapor deposition as recognized by naked eye, showing CuPc deposition (Figure 3(a)). When a part of the deposited surface was observed by SEM, interesting morphology of the deposited CuPc surface was seen. Small granular particles about 20 - $50 \mathrm{~nm}$ in size were accumulated. AFM observation revealed that height of the particles are about 10 to $15 \mathrm{~nm}$ as seen in Figure 3(c). The deposited film was cut and cross-section of the film was observed (Figure 3(d)). The small particles were grown perpendicular to the substrate. For confirmation of crystallinity of the deposited film, we measured the XRD of the film (Figure 4). Two crystal structures, $\alpha$ and $\beta$ forms, of CuPcare known [3] [4]. It is said that metastable $\alpha$ form may be transformed into stable $\beta$ form [5]. In Figure 4, XRD patterns of $\alpha$ and $\beta$ forms, calculated using reported crystallographic data, were shown in blue and green lines, respectively. Red line in Figure 4 is the measured XRD pattern of the deposited sample shown in Figures 3(a)-(d). It may be said that the XRD pattern is broad, indicating amorphous. Though only one peak is recognized in red line, broadening of the peak makes difficult to index the peak.

Depending on our accumulation of experimental data by changing the substrate temperature using another apparatus, it was found that the small spherical particles were obtained when the substrate temperature is low. On higher substrate temperature, needle-like crystalline particles were deposited on the surface to form a mesh-

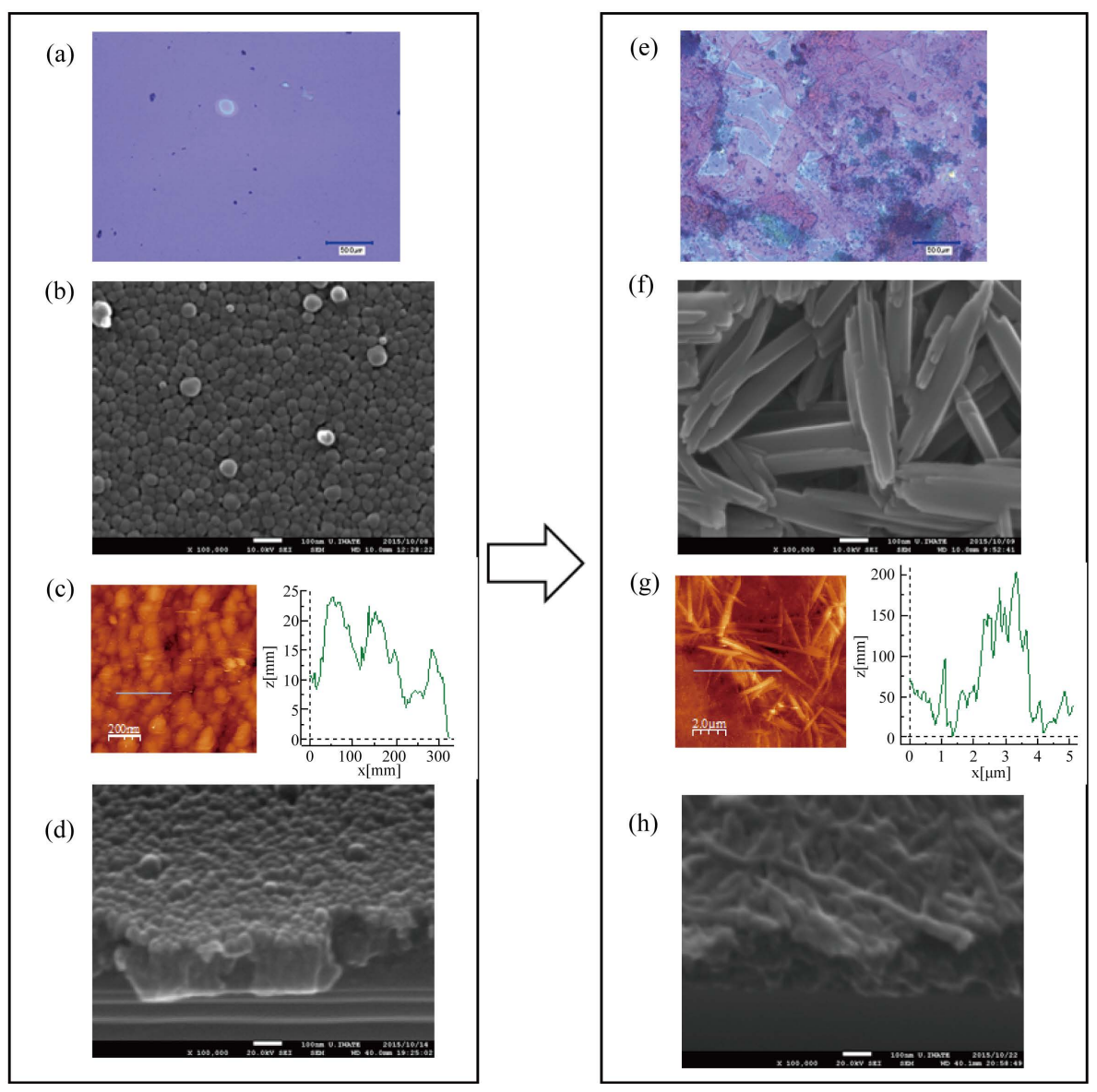

Figure 3. Microscopic pictures of CuPc solid films before and after solvent treatment. (a) Optical microscopic picture of the film after vaccum deposition; (b) SEM picture of (a); (c) AFM topographic pictures of (a); (d) cross-sectional view of (a); (e) optical microscopicture of solvent treatment of (a); (f) SEM picture of (e); (g) AFM topographic piture of (e); (h) crosssection of (e). 


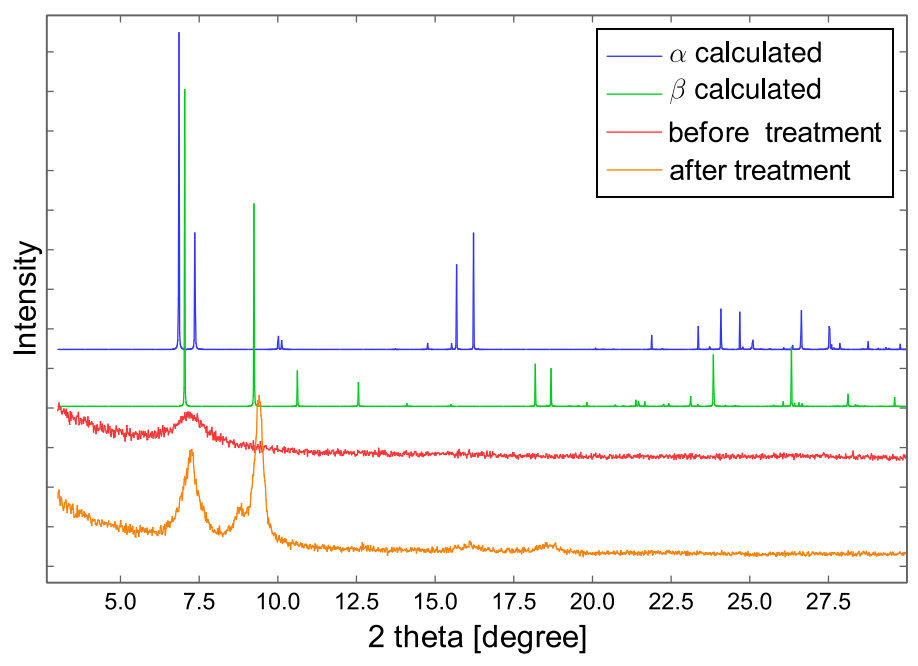

Figure 4. Powder X-ray diffraction patterns of CuPc. Blue and green lines: caluculated patterns of a form and b form, respectively. Red line: CuPc film deposited in dry process, orange line: CuPc film after wet process.

like structure. This must be caused by supercooling difference. In lower substrate temperature case, higher supercooling was generated, resulting shower of nucleation. With the increase of the substrate temperature, decrease of supercooling brought crystal growth dominant crystallization.

\subsection{Crystallization of CuPc in Wet Process}

At the second stage of the experiment, treatment of the amorphous film by the wet process was carried. Typical example is shown in Figures 3(e)-(h). When a small amount of aniline was dropped onto the film surface, change in film state was observed even by an optical microscope (Figure 3(e)). Initially flat surface (Figure 3(a)) was changed to rough. SEM observation of the rough surface revealed appearance of needle like particles (Figure 3(f)). The size of a particle, as seen in Figure 3(g), is about ten times larger than that of before aniline treatment. This is surprising result for us because CuPc is usually difficult to be desolved in a common solvent such as aniline. However, film state was changed drastically as shown in Figures 3(a)-(h). Reproducibility of our finding were recognized, thus it may be concluded as follows. Amorphousization of the deposited film make it possible to be dissolved into the solvent and when CuPc/aniline solution reached supersaturated nucleation and crystal growth of the CuPc must be occurred in the wet process.

It is well known that most organic electronic compounds are difficult to be dissolved in common solvents. Then application of film preparation by wet process, which has some advantage than dry process, has been considered difficult. However, our finding shown in this report makes the application of wet process possible for insoluble materials. Our methodology proposed in this study must be applied for organic solar cell production processes because control of the morphological structure of donor and accepter interface is important.

\section{Conclusion}

CuPc was found possible to be dissolved in common solvent such as aniline by amorphousization, and experimental evidence of growth of CuPc crystals in the solution has been shown.

\section{References}

[1] Lin, Y., Li, Y. and Zhan, X. (2012) Small Molecule Semiconductors for High-Efficiency Organic Photovoltaics. Chemical Society Reviews, 41, 4245-4272. http://dx.doi.org/10.1039/c2cs15313k

[2] Mizukami, M., Oku, S., Cho, S.-I., Tatetsu, M., Abiko, M., Mamada, M., Sakanoue, T., Suzuri, Y., Kido, J., Tokito, S. (2015) A Solution-Processed Organic Thin-Film Transistor Backplane for Flexible Multiphoton Emission Organic Light-Emitting Diode Displays. Electron Device Letters, IEEE, 36, 841-843. http://dx.doi.org/10.1109/LED.2015.2443184 
[3] Hoshino, A., Takenaka, Y. and Miyaji, H. (2003) Redetermination of the Crystal Structure of $\alpha$-Copper Phthalocyanine Grown on KCl. Acta Crystallographica, B59, 393-403. http://dx.doi.org/10.1107/s010876810300942x

[4] Brown, C.J. (1968) Crystal Structure of $\beta$-Copperphthalocyanine. Journal of the Chemical Society A, 2488-2493. http://dx.doi.org/10.1039/J19680002488

[5] Beynon, J.H. and Humphries, A.R. (1955) The Enthalpy Difference between $\alpha$ - and $\beta$-Copper Phthalocyanine Measured with an Isothermal Calorimeter. Transactions of the Faraday Society, 51, 1065-1071. http://dx.doi.org/10.1039/tf9555101065 